\title{
Dissolution of Yttrium lons and Phase Transformation of 3Y-TZP Powder in Aqueous Solution
}

\author{
Fang-Yuan Ho and Wen-Cheng J. Wei ${ }^{*}$ \\ Institute of Materials Science and Engineering, National Taiwan University, Taipei, Taiwan 106, Republic of China
}

In order to understand the reactions of yttria-containing zirconia powder in aqueous solution, the dissolution behavior of yttrium and the formation of the monoclinic $(m)$ phase of the Y-TZP powder were investigated. The tests were conducted in aqueous solution at temperatures up to $95^{\circ} \mathrm{C}$. The results showed that the reaction order of yttrium dissolution from the surface of TZP powder is 5-9, and the activation energy is about $8.4 \mathrm{~kJ} / \mathrm{mol}$. However, the value of the reaction order is different from that $(0.96-1.20)$ of the formation of $m$-phase. However, the yttrium dissolution of the slurries with the addition of 2 mol\% dispersant (D-134) was reduced greatly to an extremely low $(<2$ ppm) level up to $24 \mathrm{~h}$ at the testing temperatures. The results also showed that the amounts of $m$-phase formation were nearly the same for the powders in the slurries with or without D-134 addition. The decrease of yttrium content in the presence of $D-134$ and the formation of the $m$-phase of the TZP powder were not closely related. The degradation mechanism, or the formation of $m$-phase, should not be directly related to the dissolution of yttrium ions from a zirconia surface.

\section{Introduction}

$\mathrm{C}$ OLLOIDAL processing of fine ceramic powders has been an important method for making parts because of its uniform microstructure and strong properties. ${ }^{1}$ However, the uses of Y-doped tetragonal polycrystalline (Y-TZP) powder in aqueous suspension require further attention due to the reported yttrium dissolution. ${ }^{2}$ It has been reported that Y-TZP experiences phase transformation from tetragonal $(t)$ phase to $m$-phase and mechanical degradation when aged at 60 $400^{\circ} \mathrm{C}{ }^{3-13}$ Moreover, this phenomenon is more pronounced if the environment contains a humid atmosphere or water vapor. Therefore, the application of Y-TZP is limited to room temperature or dry conditions because of its mechanical degradation. ${ }^{9-14}$

However, the reports concerning the degradation mechanism of bulk zirconia materials offer serveral possibilities. One of the proposed possibilities is that the decrease in strength is mainly correlated to stress corrosion when the materials contain surface cracks. Sato and Shimada ${ }^{3}$ have proposed that hydroxide ions can adsorb onto the surface of zirconia materials via van der Waals forces and form $\mathrm{Zr}-\mathrm{OH}$. According to the weight change, the interaction of $\mathrm{Y}-\mathrm{TZP}$ with $\mathrm{H}_{2} \mathrm{O}$ can be expressed as follows:

$$
\begin{aligned}
&\left(\mathrm{Zr}_{1-x} \mathrm{Y}_{x}\right) \mathrm{O}_{2-x / 2} \square_{x / 2}+\delta \mathrm{H}_{2} \mathrm{O} \rightarrow \rightarrow \\
&\left(\mathrm{Zr}_{1-x} \mathrm{Y}_{x}\right) \mathrm{O}_{2-2 / x-\delta}(\mathrm{OH})_{2 \delta} \square_{x / 2-\delta}
\end{aligned}
$$

Approximately $60 \%$ of oxygen vacancies are occupied by $\mathrm{OH}^{-}$.

K. J. Bowman-contributing editor

\footnotetext{
Manuscript No. 189783. Received November 30, 1998; approved April 5, 1999.
} *Member, American Ceramic Society.
In addition, Yoshimura et al. ${ }^{4}$ have proved that hydroxide ions adsorb onto the surface of hydrothermally treated Y-TZP by using the IR technique. Additionally, Lange et al. ${ }^{5}$ have reported on the yttrium dissolution of $6.6 \mathrm{Y}-\mathrm{ZrO}_{2}$ in solution, forming $\alpha-\mathrm{Y}(\mathrm{OH})_{3}$ to a thickness of $20-50 \mu \mathrm{m}$ by TEM observation. The cubic zirconia phase where the yttria content is depleted on the surface becomes the nucleation site of the $m$-phase. The phenomenon of yttrium dissolution has also been confirmed by Thompson and Rawlings. ${ }^{6}$ However, the diffusion coefficient of $\mathrm{OH}^{-}$is faster than that of $\mathrm{O}^{2-}$ and the diffusion coefficients of both ions are faster than that of $\mathrm{Y}^{3+}$. Therefore, the dissolution of yttrium is not the main cause of the degradation of bulk TZP samples. In fact, the interaction between $\mathrm{OH}^{-}$and Y-TZP is the major reason for the degradation.

In addition, Sato et al $^{3}$ and Standard et al..$^{11}$ have concluded that the relationship between the amount of monoclinic phase and the aging time is a first-order reaction independent of the grain size of sintered TZP. According to the activation energy of the reaction, the degradation of the zirconia is controlled by an interfacial chemical reaction.

In order to understand the possible reactions of TZP powder in a suspension stage, the yttrium dissolution and the amount of monoclonic phase of the powder were quantitatively measured. The influence of the aqueous carrier on the Y-containing zirconia powder could be used to understand the degradation behavior of Y-TZP at ambient temperature.

\section{Experimental Procedure}

\section{(1) Measurement of Yttrium Dissolution}

D-134 (Dai-Ichi Kogyo Seiyaku Co., Ltd., Japan; 0 or 2 mol\%) was added to the TZP slurry (TZ3Y powder, Tosoh Co., Japan) as a dispersant. D-134 is an ammonium salt homopolymer with a 2-propenoic acid group. These slurries were milled for $10 \mathrm{~min}$, then sealed in a glass tube and aged at $25^{\circ}$ to $95^{\circ} \mathrm{C}$ for 3 to $24 \mathrm{~h}$. The aged powder and the clear supernatant solution were separated using a centrifugal technique. The clear solution was sampled to test for the yttrium concentration (ICP, AtomScan 16, Thermal Jarrell Ash Co., USA).

Prior to the measurement of the yttrium content, a series of yttrium standard solutions containing $0,25,50,100$, or 200 ppm yttrium were used for the calibration of spectrum intensity. The calibration curve revealed a good linear relationship within $200 \mathrm{ppm}$ concentration. ${ }^{17}$ In order to avoid spectrum shift, the slurry containing $100 \mathrm{ppm}$ yttrium was used for regular calibration every four measurements.

\section{(2) Phase Transformation of TZP}

The powder obtained from centrifugal treatment was dried at $105^{\circ} \mathrm{C}$ and milled to pass 120 mesh. The change of $\mathrm{m}$-phase content was quantitatively determined by using X-ray diffractometry (XRD, PW1792, Philips Instruments, Netherlands) according to the procedure given by Toraya et al. ${ }^{15}$ and Garvie et $a l .{ }^{16}$ The XRD was operated at $40 \mathrm{kV}$ and $30 \mathrm{~mA}$. The quantity of $m$-phase formation could be measured with a reproducibility of $3 \%$. 


\section{Results and Discussion}

\section{(1) Yttrium Dissolution}

The amount of yttrium content in the TZP slurry with 0 or 2 mol\% D-134 is shown in Fig. 1, which is a plot of the amount of yttrium dissolution, $\ln [-\ln (1-C)]$, versus the aging time, $\ln t$. This amount increases exponentially with increasing aging time. Furthermore, the saturation concentration of the yttrium decreases as the aging temperature decreases. The best fitting equations are shown in Fig. 1 at various aging temperatures. As seen in Fig. 1 the dissolved yttrium content in the slurry is low when aged at $25^{\circ} \mathrm{C}$. In addition, the yttrium content of the slurry with 2 mol\% D-134 is extremely low when aged at $25-95^{\circ} \mathrm{C}$ (Fig. 2). Furthermore, the aging temperature shows no significant effect on the amount of yttrium dissolution. The interaction of D-134 with the TZP powder surface might interfere with the dissolution behavior of yttrium in aqueous solution.

When the slurries without D-134 addition have yttrium dissolution, a diffusion model of yttrium from the surface of TZP particles can be considered. The kinetics of the yttrium dissolution can be expressed as follows:

$$
\begin{aligned}
& C=1-\exp \left(-k_{1} t^{n}\right) \\
& 1-C=\exp \left(-k_{1} t^{n}\right)
\end{aligned}
$$

where $C$ is the concentration of yttrium ions in the solution, $t$ is the aging time (hours), $k_{1}$ is the reaction constant, and $1 / n$ is the reaction order. After calculating the natural logarithm twice, Eq. (2) becomes

$$
\ln [-\ln (1-C)]=n \ln t+\ln k_{I}
$$

From the above equations the reaction order $(1 / n)$ of the yttrium dissolution is from 5 to 9 with or without D-134. Moreover, the activation energy can be calculated based on the Arrhenius equation,

$$
\ln k_{1}=\left[-Q_{1} / R T\right]+B
$$

where $Q_{1}$ is the activation energy $(\mathrm{J} / \mathrm{mol}), R$ is the gas constant $(8.314 \mathrm{~J} /(\mathrm{mol} \cdot \mathrm{K}))$, and $B$ is a constant. When the $\ln k_{1}$ value obtained was plotted against inverse aging temperature, the activation energy obtained was about $8.4 \mathrm{~kJ} / \mathrm{mol}$, as shown in Table I.

\section{(2) Formation of Monoclinic Phase}

Figure 3 is the $\mathrm{X}$-ray diffraction spectra of as-received and aged TZP powders at different temperatures. The quantity of

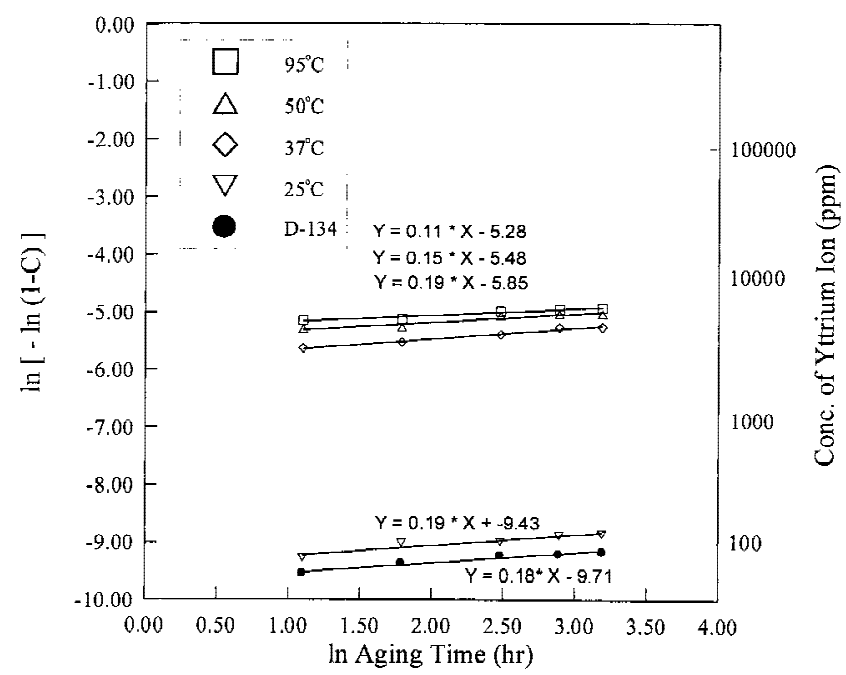

Fig. 1. Yttrium concentration in the suspension as a function of aging temperature and time. The sample with D-134 was tested at $37^{\circ} \mathrm{C}$.

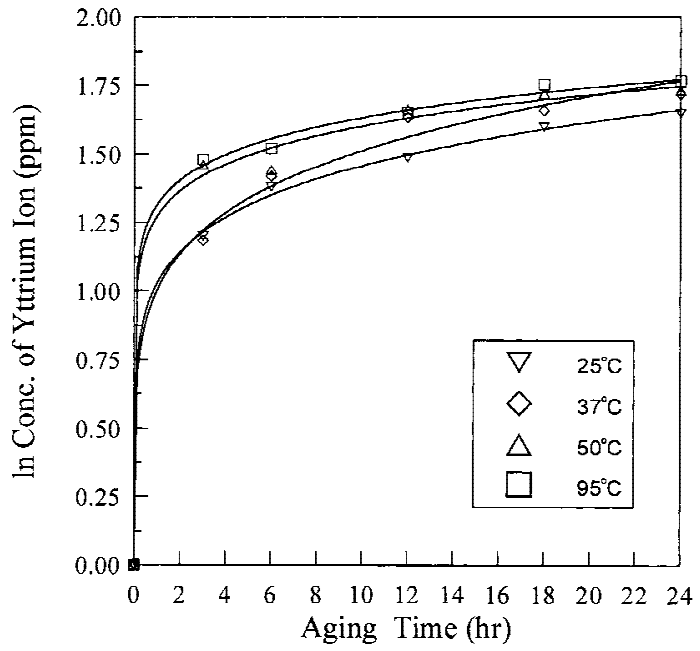

Fig. 2. Yttrium concentration in the suspension with 2 mol\% D-134 at various temperatures plotted against aging time.

Table I. Values of $n, 1 / n$, Reaction Constant $\left(k_{1}\right)$, and Activation Energy $\left(Q_{1}\right)$ for Yttrium Dissolution from the Surface of TZP Powder to Aqueous Solution

\begin{tabular}{ccccc}
\hline $\begin{array}{c}\text { Aging } \\
\text { condition }\left({ }^{\circ} \mathrm{C}\right)\end{array}$ & $n$ & $1 / n$ & $k_{1}$ & $\begin{array}{c}Q_{1} \\
(\mathrm{~kJ} / \mathrm{mol})\end{array}$ \\
\hline 95 & 0.11 & 9.1 & $5.0 \times 10^{-3}$ & \\
50 & 0.15 & 6.7 & $4.1 \times 10^{-3}$ & \\
37 & 0.19 & 5.3 & $2.7 \times 10^{-3}$ & \\
25 & 0.18 & 5.6 & $0.80 \times 10^{-4}$ & 8.4 \\
\hline
\end{tabular}

the $m$-phase in the $95^{\circ} \mathrm{C} / 6$-h-aged powder is obviously greater than the as-received. Suppose the amount of monclinic phase can be expressed as follows:

$$
M=1-\exp \left(-k_{2} t^{m}\right)
$$

where $M$ is the amount of $m$-phase, $k_{2}$ is the reaction constant, and $1 / m$ is the reaction order of the transformation. After calculating the natural logarithm twice, Eq. (5) becomes

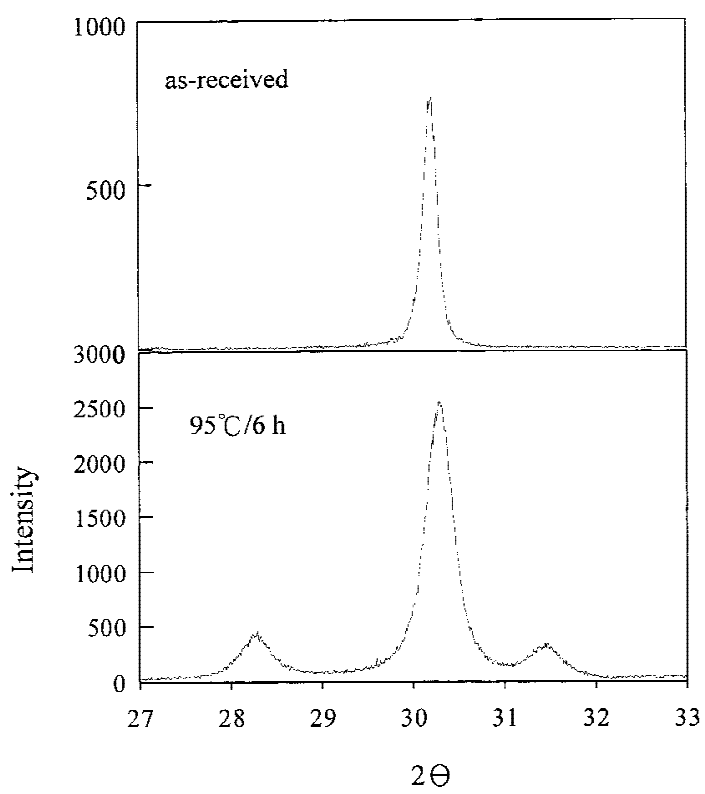

Fig. 3. X-ray spectra of TZP powders either as-received or aged in $95^{\circ} \mathrm{C}$ aqueous solution for $6 \mathrm{~h}$. 
$\ln [-\ln (1-M)]=m \ln t+\ln k_{2}$

The best fit equations are shown in Fig. 4 at each testing temperature. From the figure the reaction order $1 / \mathrm{m}$ is from 0.96 to 1.20 for $m$-phase transformation of YTZP powder. The amount of $m$-phase formed during the aging test increases exponentially with increasing aging time. Also the amount of the $m$-phase increases with aging temperature. The percentage of the $m$-phase may increase to $68 \%$ if the aging conditions last for $24 \mathrm{~h}$ at $95^{\circ} \mathrm{C}$. When comparing two slurries with 0 or 2 mol\% D-134 addition and aging at $37^{\circ} \mathrm{C}$, the transformed $\mathrm{m}$ phase of these TZP powders is almost identical.

The values of $k_{2}$ at each testing temperature obtained have also been plotted against inverse aging temperature. The results obtained by Shimuzu et $a l .{ }^{14}$ and Sato et $a l .{ }^{3}$ are also shown in Fig. 5. The activation energy $Q_{2}$ is obtained from the slope of the best fit lines in Fig. 5. The activation energy $Q_{2}$ of this test is $36.2 \mathrm{~kJ} / \mathrm{mol}$ and is close to but not equal to that obtained in the literature..$^{3,14}$ The value of the reaction order and the activation energy reveal that the transformation reaction is interfacial control through the diffusion of $\mathrm{OH}^{-}$molecules into the TZP surface. ${ }^{12}$

Some research ${ }^{5,6}$ suggests that the yttrium dissolution of zirconia materials, causing local phase instability, leads to the formation of monoclinic phase. In fact, the dissolution of yttrium ions is retarded by the use of D-134 dispersant. The results that we obtained show different trends in yttrium dissolution and $m$-phase formation. Therefore, it can be concluded that yttrium dissolution is not the controlling factor leading to $m$-phase formation.

\section{Conclusion}

(1) During colloidal processing, TZP powder with a high specific surface area undergoes yttrium dissolution and the formation of $m$-phase.

(2) The reaction order $1 / n$ of yttrium dissolution from the surface of TZP powder to aqueous solution is between 5 and 9 and the activation energy is about $8.5 \mathrm{~kJ} / \mathrm{mol}$. If the slurry contains $2 \mathrm{~mol} \% \mathrm{D}-134$, the amount of yttrium dissolution is extremely low (<2 ppm). The fixation of D-134 for yttrium on the surface of TZP powder is evident.

(3) The amount of monoclinic phase increases exponentially with increasing aging time and temperature. The results

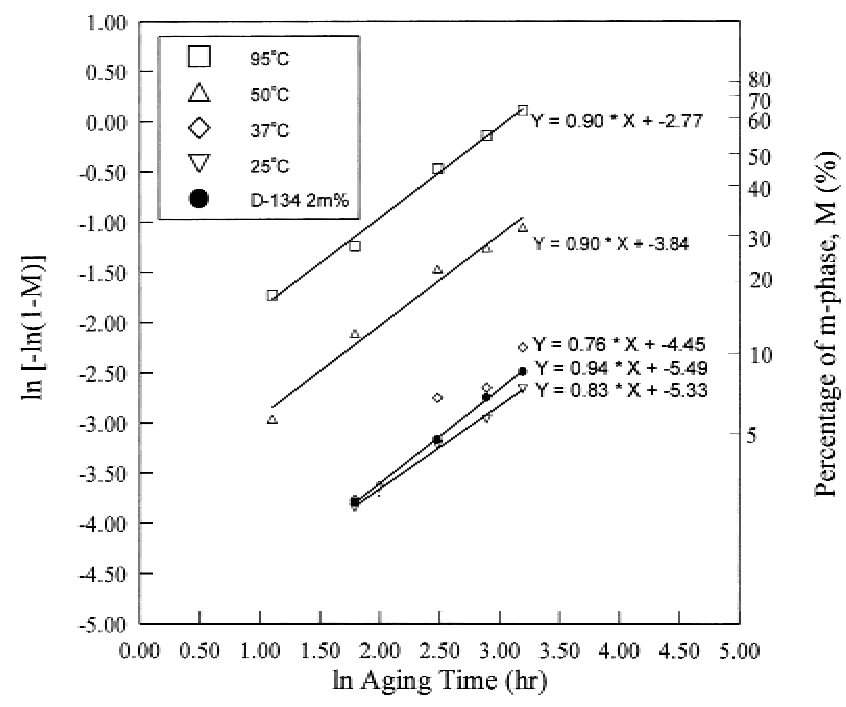

Fig. 4. Arrhenius plot of the reaction constant $K_{2}$ as a function of $1 / T$. The sample containing D-134 was tested at $37^{\circ} \mathrm{C}$.

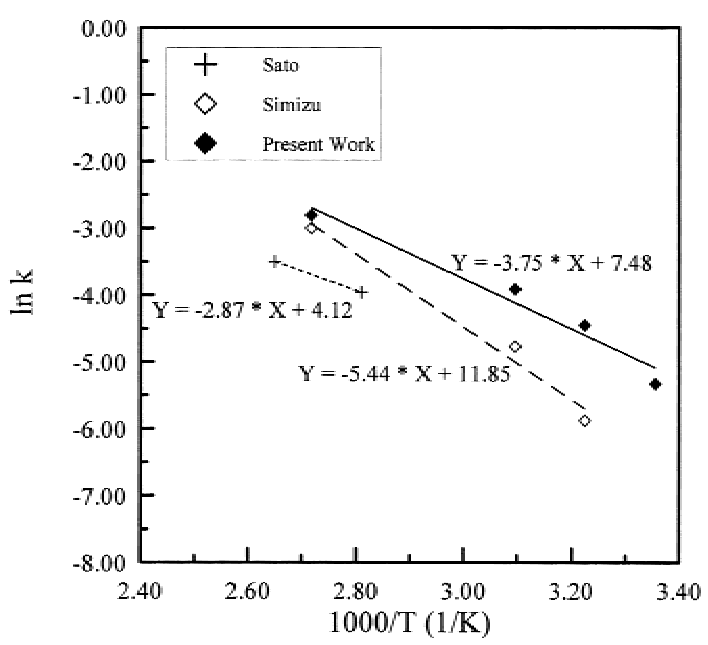

Fig. 5. Arrhenius plot of the reaction constant $k$ of yttrium dissolution for Y-TZP powder in water.

show that the reaction order $1 / m$ to form $m$-phase in aqueous solution is from 0.96 to 1.20 and the activation energy is approximately $36.16 \mathrm{~kJ} / \mathrm{mol}$. This is consistent with the reaction kinetics controlled by the interfacial reaction induced by water molecules.

(4) The addition of D-134 cannot alter the transformation of $m$-phase. This phenomenon contradicts the results of yttrium dissolution.

Acknowledgments: We are indebted to the National Science Council for financial support (NSC86-2622-E002-026R) and to Mr. J. L. Shen at the Taiwan Provincial Handicraft Research Institute for the ICP analysis.

\section{References}

${ }^{1}$ I. A. Aksay, F. F. Lange, and B. I. Davis, "Uniformity of $\mathrm{Al}_{2} \mathrm{O}_{3}-\mathrm{ZrO}_{2}$ Composites by Colloidal Filtration," J. Am. Ceram. Soc., 66 [10] C-190-C-192 (1983).

${ }^{2}$ M. Yasrebi, M. Ziomek-Moroz, W. Kemp, and D. H. Sturgis, "Role of Particle Dissolution in the Stability of Binary Yttria-Silica Colloidal Suspension," J. Am. Ceram. Soc., 79 [5] 1223-27 (1996).

${ }^{3}$ T. Sato and M. Shimada, "Transformation of Yttria-Doped Tetragonal $\mathrm{ZrO}$ Polycrystals by Annealing in Water," J. Am. Ceram. Soc., 68 [6] 356-59 (1985).

${ }^{4} \mathrm{M}$. Yoshimura and T. Noma, "Role of $\mathrm{H}_{2} \mathrm{O}$ on the Degradation Process of Y-TZP," J. Mater. Sci. Lett., 6, 455-67 (1987).

${ }^{5}$ F. F. Lange, B. I. Davis, and G. L. Dunlop, "Degradation during Aging of Transformation Toughened $\mathrm{ZrO}_{2}-\mathrm{Y}_{2} \mathrm{O}_{3}$ Materials at $250^{\circ} \mathrm{C}$," J. Am. Ceram. Soc., 69 [4] 237-40 (1986).

${ }^{6}$ I. Thompson and R. D. Rawlings, "Effects of Liquid Environments on Zirconia-Toughened Alumnia," J. Mater. Sci., 27, 2823-30 (1992).

${ }^{7}$ L. J. Kook, "Microstructural Development of the Isothermal Phase Transformation during Aging at $250^{\circ} \mathrm{C}$ in 2 Y-TZP Ceramics," J. Mater. Sci., 29 , 136-40 (1994).

${ }^{8}$ S. Lawson, "A New Technique for Monitoring Aging in Yttria-Tetragonal Zirconia Polycrystals," J. Am. Ceram. Soc., 76 [12] 3170-72 (1993).

${ }^{9}$ T. Sato and M. Shimada, "Crystalline Phase Change in Yttria-Partially Stabilized Zirconia by Low-Temperature Annealing," J. Am. Ceram. Soc., 10, C-212 (1984).

${ }^{10}$ J. L. Drummond, "In Vitro Aging of Yttria-Stabilized Zirconia," J. Am. Ceram. Soc., 72 [4] 675-76 (1989).

${ }^{11}$ O. C. Standard and K. Schindhelm, "In-Vitro Aging of Tetragonal Zirconia Polycrystal Ceramics," J. Aust. Ceram. Soc., 29 [1/2] 111-23 (1993).

${ }^{12} \mathrm{M}$. Hirano, "Inhibition of Low Temperature Degradation of Tetragonal Zirconia Ceramics-A Review,” Br. Ceram. Trans. J., 91, 139-47 (1992).

${ }^{13}$ L. K. Matsumoto, "Strength Recovery in Degraded Yttria-Doped Tetragonal Zirconia Polycrystals," J. Am. Ceram. Soc., 68 [8] C-213 (1985).

${ }^{14}$ K. Shimizu, M. Oka, P. Kumar, Y. Kotoura, K. Makinouch, and T. Nakamura, "Time-Dependent Changes in the Mechanical Properties of Zirconia Ceramics," J. Biomed. Mater. Res., 27, 729-34 (1993).

${ }^{15} \mathrm{H}$. Toraya, M. Yoshimura, and S. Somiya, "Calibration Curve for Quantitative Analysis of the Monoclinic-Tetragonal $\mathrm{ZrO}_{2}$ System by X-ray Diffraction," J. Am. Ceram, Soc., 67 [6] C-119-C-121 (1984).

${ }^{16}$ R. Garvie and P. S. Nicholson, "Phase Analysis in Zirconia Systems," J. Am. Ceram. Soc., 55 [6] 303-305 (1972).

${ }^{17}$ F. Y. Ho; Master's Thesis. National Taiwan University, Taipei, Taiwan, June 1998 